\title{
Ground Glass Opacity with Mixed Consolidation on Chest Computed Tomography Reflects the Severe Condition of Pneumocystis Pneumonia in Association with a Poor Prognosis in Patients with Connective Tissue Diseases
}

\author{
Mitsuhiro Akiyama, Yuko Kaneko and Tsutomu Takeuchi
}

\begin{abstract}
:
Objective Pneumocystis pneumonia (PCP) is a serious fungal infection that can be life threatening in immunocompromised hosts. We evaluated the association between the radiological patterns of PCP on highresolution computed tomography (HRCT) and clinical characteristics and the prognosis of patients with connective tissue disease (CTD).

Methods All CTD patients who developed PCP from January 1999 to April 2017 were retrospectively evaluated. Patients were divided into three groups based on their chest HRCT findings: Ground glass opacity (GGO) sharply demarcated from the adjacent normal lung by interlobular septa (demarcated GGO), diffuse GGO without obvious demarcation (diffuse GGO), and GGO with mixed consolidation (mixed GGO). We compared the clinical characteristics at the onset of PCP and the outcomes among the groups.

Results A total of 35 cases were identified: demarcated GGO ( $\mathrm{n}=8,23 \%)$, diffuse GGO $(\mathrm{n}=19,54 \%)$, and mixed GGO $(n=8,23 \%)$. The mixed GGO group showed a higher serum C-reactive protein level $(\mathrm{p}<0.0001)$, lower lymphocyte count $(\mathrm{p}=0.07)$, lower serum albumin $(\mathrm{p}<0.001)$, and lower partial pressure of arterial oxygen/fraction of inspiratory oxygen ratios $(\mathrm{p}<0.001)$ in comparison to the demarcated and diffuse GGO groups. The mixed GGO group showed significantly higher mortality in comparison to the demarcated and diffuse GGO groups ( $88 \%$ vs. $7 \%, \mathrm{p}<0.0001)$.

Conclusion GGO with mixed consolidation on chest HRCT was associated with a poor outcome of PCP in patients with CTD.
\end{abstract}

Key words: pneumocystis pneumonia, mortality, connective tissue disease, high-resolution computed tomography, consolidation

(Intern Med 58: 3379-3383, 2019)

(DOI: 10.2169/internalmedicine.3182-19)

\section{Introduction}

Pneumocystis pneumonia (PCP) is a serious infection that causes immense inflammation of the lungs with potentially fatal consequences in immunocompromised hosts such as patients with human-immunodeficiency virus (HIV) infection or patients receiving immunosuppressive treatment $(1,2)$. PCP is also a common opportunistic condition in patients with connective tissue diseases (CTDs), and the resultant respiratory failure is more severe in such patients than in those infected with HIV (3). High-resolution computed tomography (HRCT) of the lungs is an essential tool that reveals various findings in the diagnosis and assessment of PCP. However, the condition can be classified into three patterns based on the distribution of ground-glass opacity (GGO) (4) as GGO sharply demarcated from the adjacent normal lung by interlobular septa, diffuse GGO without obvious demarcation, and GGO with mixed consolidation. The distinct patterns have been suggested to reflect different pathogenic reactions to Pneumocystis jiroveci. Nonetheless, little is known about the frequency or the clinical features of 


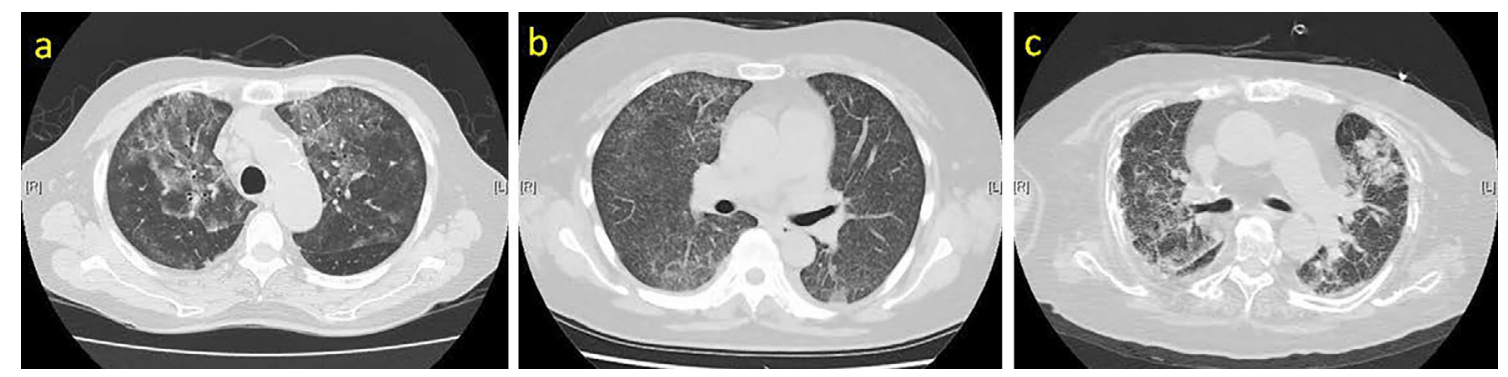

Figure. Representative findings of three patterns of PCP detected on high resolution computed tomography. (a) GGO sharply demarcated from the adjacent normal lung by interlobular septa (Demarcated GGO), (b) Diffuse GGO without obvious demarcation (Diffuse GGO), (c) GGO with mixed consolidation (Mixed GGO). PCP: pneumocystis pneumonia, GGO: ground glass opacity

PCP according to each radiological type in patients with CTD. In the present study, we evaluated the association between the radiological patterns of PCP on chest HRCT; and the clinical characteristics and prognosis of patients with CTD.

\section{Materials and Methods}

We retrospectively reviewed all consecutive patients who developed PCP during immunosuppressive treatment for underlying CTDs at our institution from January 1999 to April 2017. Definite PCP was defined based on the criteria outlined in a previous report (5), based on the fulfillment of the following criteria: (i) clinical respiratory manifestations, (ii) GGO on chest HRCT, (iii) microscopic detection of Pneumocystis jiroveci or positive polymerase-chain-reaction test results for Pneumocystis jiroveci deoxyribonucleic acid in respiratory specimens, (iv) increased serum $\beta$-D glucan levels (turbidimetric kinetic method, Wako Pure Chemical Industries, Tokyo, Japan), and (v) exclusion of other types of infectious pneumonitis caused by bacteria, viruses, or fungi. The presence of other types of infectious pneumonitis was determined by each attending physician based on sputum and blood cultures, urinary/blood antigens, and/or antibody measurement.

The demographic characteristics and laboratory findings at the onset of PCP were collected from patient charts. Chest HRCT findings were evaluated by two investigators (MA and $\mathrm{YK}$ ) based on the radiologist's radiograph interpretation report. Chest HRCT findings were classified into three patterns described in a previous study (4): GGO sharply demarcated from the adjacent normal lung by interlobular septa (demarcated GGO), diffuse GGO without obvious demarcation (diffuse GGO), and demarcated or diffuse GGO with mixed consolidation (mixed GGO) (Figure). There was consensus among the two investigators regarding the classification of the patterns. The clinical characteristics and prognosis of the included patients were investigated.

The Ethics Committee of Keio University School of Medicine approved this study. The requirement for written informed consent was waived based on Japanese guidelines. All investigations were conducted according to the princi- ples of the Declaration of Helsinki.

\section{Statistical analyses}

Continuous variables were compared using Student's $t$ test. Categorical variables were analyzed by the chi-squared test. $\mathrm{P}$ values (two-sided) of $<0.05$ were considered to indicate statistical significance. All statistical analyses were performed using the EZR software program (Saitama Medical Center, Jichi Medical University, Saitama, Japan) (6).

\section{Results}

A total of 35 patients with PCP were identified and divided into three groups based on the HRCT findings as follows: demarcated GGO $(n=8,23 \%)$, diffuse GGO $(n=19$, $54 \%)$, and mixed GGO $(\mathrm{n}=8,23 \%)$. The patient demographics at the onset of PCP are presented in Table. No patient was under prophylaxis with trimethoprim-sulfamethoxazole against PCP. The underlying connective tissue diseases included rheumatoid arthritis $(\mathrm{n}=19,54 \%)$, systemic lupus erythematosus $(n=9,26 \%)$, vasculitis $(n=6,17 \%)$, and mixed connective tissue disease $(n=1,3 \%)$. The prevalence of underlying connective tissue diseases and their treatments, including glucocorticoids, biologic agents, methotrexate, and other immunosuppressants did not differ among the demarcated GGO, diffuse GGO, and mixed GGO groups. Furthermore, there were no significant differences among the three groups in the incidence of comorbidities such as renal dysfunction, lung disease, cardiac disease, and liver dysfunction.

When patients of the non-mixed GGO (demarcated and diffuse GGO) groups were compared with those of the mixed GGO group, we found a significant difference in the clinical and laboratory findings. The presentation of PCP was more acute in patients with a mixed GGO pattern than in those without (duration from the onset to the diagnosis: 5 days vs. 13 days, $\mathrm{p}=0.06$ ). Laboratory tests showed that the mixed GGO group had a higher serum C-reactive protein level $(17.1 \mathrm{mg} / \mathrm{dL}$ vs. $4.5 \mathrm{mg} / \mathrm{dL}, \mathrm{p}<0.0001)$, lower lymphocyte count $(379 / \mu \mathrm{L}$ vs. $802 / \mu \mathrm{L}, \mathrm{p}=0.07)$, lower serum albumin level $(2.1 \mathrm{~g} / \mathrm{dL}$ vs. $3.0 \mathrm{~g} / \mathrm{dL}, \mathrm{p}<0.001)$, and lower partial pressure of arterial oxygen $\left(\mathrm{PaO}_{2}\right) /$ fraction of inspiratory 
Table. Comparison of the PCP Patients Stratified by the Radiological Findings.

\begin{tabular}{|c|c|c|c|c|c|}
\hline \multirow[b]{2}{*}{ Characteristics } & \multicolumn{3}{|c|}{ Non-mixed GGO n=27 } & \multirow[b]{2}{*}{$\begin{array}{c}\text { Mixed } \\
\text { GGO } \\
\mathrm{n}=8\end{array}$} & \multirow[b]{2}{*}{$\begin{array}{c}\text { p value } \\
\text { (Mixed } \\
\text { GGO vs. } \\
\text { non-mixed } \\
\text { GGO) }\end{array}$} \\
\hline & $\begin{array}{c}\text { Demarcated } \\
\text { GGO } \\
n=8\end{array}$ & $\begin{array}{c}\text { Diffuse } \\
\text { GGO } \\
n=19\end{array}$ & $\begin{array}{c}\text { Total } \\
\text { non-mixed } \\
\text { GGO } \\
\mathrm{n}=27\end{array}$ & & \\
\hline Age, years, mean $(\mathrm{SD})$ & $60(15)$ & $65(14)$ & $63(14)$ & $65(18)$ & ns \\
\hline Female, n $(\%)$ & $8(100)$ & $10(53)$ & $18(67)$ & $6(75)$ & ns \\
\hline Duration from onset of symptoms to diagnosis, days, mean (SD) & $12(10)$ & $13(11)$ & $13(11)$ & $5(3)$ & 0.06 \\
\hline Smoking, n (\%) & $1(13)$ & $7(37)$ & $8(30)$ & $1(13)$ & ns \\
\hline Underlying disease, $\mathrm{n}(\%)$ & & & & & ns \\
\hline Rheumatoid arthritis & $4(50)$ & $12(63)$ & $16(59)$ & $3(38)$ & \\
\hline Systemic lupus erythematosus & $3(38)$ & $4(21)$ & $7(26)$ & $2(25)$ & \\
\hline Vasculitis & $0(0)$ & $3(16)$ & $3(11)$ & $3(38)$ & \\
\hline Mixed connective tissue disease & $1(13)$ & $0(0)$ & $1(4)$ & $0(0)$ & \\
\hline \multicolumn{6}{|l|}{ Comorbidity, n (\%) } \\
\hline Renal dysfunction & $4(50)$ & $11(58)$ & $15(56)$ & $4(50)$ & ns \\
\hline Lung disease ${ }^{\text {蛙 }}$ & $2(25)$ & $7(37)$ & $9(33)$ & $4(50)$ & ns \\
\hline Cardiac disease & $2(25)$ & $0(0)$ & $2(7)$ & $2(25)$ & ns \\
\hline Liver dysfunction & $5(50)$ & $3(16)$ & $8(30)$ & $5(63)$ & ns \\
\hline \multicolumn{6}{|l|}{ Treatment for CTD } \\
\hline Glucocorticoids, n (\%) & $5(63)$ & $16(84)$ & $21(78)$ & $8(100)$ & ns \\
\hline Dose of prednisolone (mg/day), mean (SD) & $17(22)$ & $13(14)$ & $14(16)$ & $20(9)$ & $\mathrm{ns}$ \\
\hline Biologic agents, $\mathrm{n}(\%)$ & $1(13)$ & $7(37)$ & $8(30)$ & $1(13)$ & ns \\
\hline Methotrexate, n (\%) & $4(50)$ & $10(53)$ & $14(52)$ & $3(38)$ & ns \\
\hline Other immunosuppressants, $\mathrm{n}(\%)$ & $4(50)$ & $8(42)$ & $12(42)$ & $3(38)$ & ns \\
\hline \multicolumn{6}{|l|}{ Laboratory findings } \\
\hline $\mathrm{PaO}_{2} / \mathrm{FiO}_{2}$ ratio, mean $(\mathrm{SD})$ & $281(94)$ & $348(88)$ & $328(93)$ & $177(87)$ & $<0.001$ \\
\hline Lymphocytes $(/ \mu \mathrm{L})$, mean $(\mathrm{SD})$ & $773(835)$ & $814(484)$ & $802(592)$ & $379(459)$ & 0.07 \\
\hline Albumin, g/dL, mean (SD) & $2.9(0.4)$ & $3.0(0.6)$ & $3.0(0.6)$ & $2.1(0.3)$ & $<0.001$ \\
\hline $\mathrm{LDH}, \mathrm{U} / \mathrm{L}$, mean $(\mathrm{SD})$ & 585 (399) & $366(193)$ & $431(281)$ & $483(110)$ & ns \\
\hline CRP, mg/dL, mean (SD) & $3.6(2.5)$ & $4.9(4.7)$ & $4.5(4.1)$ & $17.1(10.7)$ & $<0.0001$ \\
\hline $\mathrm{IgG}, \mathrm{mg} / \mathrm{dL}$, mean (SD) & $1,122(242)$ & $939(432)$ & 994 (389) & $914(616)$ & ns \\
\hline KL-6, U/mL, mean (SD) & $1,240(884)$ & $991(695)$ & $1,067(746)$ & $1,039(697)$ & ns \\
\hline$\beta$-D glucan, $\mathrm{pg} / \mathrm{mL}$, mean (SD) & 149 (137) & $215(191)$ & $195(177)$ & $201(166)$ & $\mathrm{ns}$ \\
\hline \multicolumn{6}{|l|}{ Treatment regimen for PCP } \\
\hline Pulse or high dose therapy of glucocorticoids, $\mathrm{n}(\%)$ & $6(75)$ & $13(68)$ & $19(70)$ & $8(100)$ & ns \\
\hline High dose of TMP-SMZ (15-20 mg/kg/day of TMP), $\mathrm{n}(\%)$ & $4(50)$ & $8(42)$ & $12(44)$ & $5(63)$ & ns \\
\hline Death, n $(\%)$ & $2(25)$ & $0(0)$ & $2(7)$ & $7(88)$ & $<0.0001$ \\
\hline
\end{tabular}

$\$ \mathrm{eGFR}<60 \mathrm{~mL} / \mathrm{min} / 1.73 \mathrm{~m}^{2} . \$$ Lung disease: interstitial lung disease, bronchiolitis, and chronic obstructive pulmonary disease. $\$ \$$ Cardiac disease: the past history of heart failure, ischemic heart disease, and heart amyloidosis. $\$+\$$ Liver dysfunction: alanine aminotransferase is over 37 IU/L. PCP: pneumocystis pneumonia, CTD: connective tissue disease, GGO: ground glass opacity, SD: standard deviation, ns: non-significant, KL-6: Krebs von den Lungen-6, LDH: lactate dehydrogenase, CRP: C-reactive protein, TMP-SMZ: trimethoprim-sulfamethoxazole

oxygen $\left(\mathrm{FiO}_{2}\right)$ ratio $(177$ vs. $328, \mathrm{p}<0.001)$ in comparison to the other groups.

No significant differences were observed in the other demographic characteristics of the non-mixed and mixed GGO groups, including age (65 years vs. 63 years, $\mathrm{p}=0.83$ ), Krebs von den Lungen-6 level (1,039 U/mL vs. 1,067 U/ $\mathrm{mL}, \mathrm{p}=0.95)$, lactate dehydrogenase level (483 U/L vs. 431 $\mathrm{U} / \mathrm{L}, \mathrm{p}=0.62)$, and $\beta-\mathrm{D}$ glucan level (201 pg/mL vs. $195 \mathrm{pg} /$ $\mathrm{mL}, \mathrm{p}=0.94)$.

Among the eight patients with mixed GGO, seven patients satisfied the Berlin definition criteria for acute respiratory distress syndrome (ARDS) (7). Consolidation was distributed predominantly in a dorsal location in seven of the eight cases of mixed GGO. Of note, patients with mixed
GGO pattern showed a significantly higher mortality rate in comparison to those with a non-mixed GGO pattern $(88 \%$ vs. 7\%, p<0.0001). The treatment regimens for PCP (e.g., steroid pulse or high-dose glucocorticoids and high-dose trimethoprim-sulfamethoxazole) did not differ between the mixed and non-mixed GGO groups.

The mortality rate was $21 \%$ in rheumatoid arthritis (4/19), $50 \%$ in vasculitis $(3 / 6)$, and $22 \%$ in systemic lupus erythematosus $(2 / 9)$. Of the nine deaths, three $(33 \%)$ were in patients with preexisting respiratory involvement (one rheumatoid arthritis patient with interstitial lung disease, one vasculitis patient with interstitial lung disease, and one vasculitis patient with chronic obstructive pulmonary disease). 


\section{Discussion}

In the present study, we found that the presence of GGO with consolidation was associated with a higher serum Creactive protein level, a lower serum albumin level and a lower $\mathrm{PaO}_{2} / \mathrm{FiO}_{2}$ ratio in comparison to GGO without consolidation. We also found that patients showing GGO with consolidation were more likely to have fatal outcomes in comparison to those without consolidation. Our results suggest that the radiological finding of GGO with consolidation on HRCT reflects a more severe condition and a poor prognosis of PCP in patients with CTDs.

Our study showed that diffuse GGO was the main HRCT finding (54\%) of PCP in CTD patients, which is in line with a previous report (8). Kanne et al. reported that diffuse GGO was the principal finding of PCP and reflected intraalveolar fibrin, debris, and the accumulation of the organism (8). We also demonstrated that PCP in CTD patients can present with consolidation on HRCT and that this was associated with a poor prognosis. These findings validated the recent studies that reported consolidation and crazy-paving pattern as poor prognostic radiological findings in non-HIV patients $(9,10)$. It would have been interesting to investigate the effect of the extent of consolidation area on the prognosis of PCP in CTD patients; however, the sample size of our study was too small for such an investigation. Future studies that semi-quantitatively evaluate consolidation will promote the more detailed stratification of the prognosis.

Several hypotheses could explain the mechanism of association between mixed GGO and increased severity such as rapid progression, a higher level of serum $\mathrm{C}$-reactive protein level, and a lower $\mathrm{PaO}_{2} / \mathrm{FiO}_{2}$ ratio, along with a higher mortality rate. First, a stronger neutrophilic inflammatory response against Pneumocystis jiroveci might be unleashed in patients with mixed GGO than in those without. A study on clinicopathological comparisons between HIV and non-HIV PCP patients reported that non-HIV patients had more intensive neutrophilic inflammation in their bronchoalveolar lavage fluid, despite having lower amounts of Pneumocystis jiroveci; however, the underlying pathogenesis remains unclear (11). The study also showed that in non-HIV patients with PCP, the clinical course was more acute and severe, and that the prognosis was worse (11). Importantly, another study demonstrated that consolidation on HRCT was observed in half of non-HIV PCP patients but no HIV patients with PCP (12). These studies suggest the possibility that consolidation on HRCT is associated with more neutrophilic inflammation in the lungs and a poor prognosis. Thus, we assumed that more intense neutrophilic inflammation against Pneumocystis jiroveci in the lungs occurred in the mixed GGO group in comparison to the non-mixed GGO group in the present study. A second hypothesis is that consolidation occurs due to ARDS. Patients in the present study with consolidation had lower serum albumin levels, which is consistent with the previous studies that consider low levels to be a prognostic factor for PCP (13) and a factor predicting the severity of ARDS (14). Assuming that hypoalbuminemia, severe respiratory failure, and dense opacification on HRCT are likely to represent severe edema and protein exudation within the interstitium and alveoli without cardiac failure, the poor prognosis observed in cases of mixed GGO might be ascribed to a severe condition similar to ARDS. In fact, the majority of cases in the mixed GGO group satisfied the Berlin definition criteria for ARDS. In accordance with the treatment of ARDS-although not yet established-patients with PCP with consolidation on HRCT should immediately receive intensive treatment with proper breathing support, nutritional supply, and measures to prevent secondary infection in addition to receiving treatment for PCP. The possibility of co-infection with other pathogens also remained; however, we excluded patients in whom infections other than PCP had been clinically recognized. Since the pathogenesis and mechanisms underlying the association between consolidation and a poor prognosis remain unclear, the accumulation of cases and relevant studies is needed.

The present study was associated with some limitations, including its retrospective design and the relatively small study population. Thus, larger prospective studies are needed to confirm our results and establish effective treatment protocols for such patients in order to improve their outcomes.

\section{Author's disclosure of potential Conflicts of Interest (COI).}

Mitsuhiro Akiyama: Advisory role, Cure Grades and Eisai; Honoraria, Cure Grades and Eisai. Yuko Kaneko: Honoraria, AbbVie, Astellas, Ayumi, Bristol-Myers Squibb, Chugai, Eisai, Eli Lilly, Hisamitsu, Jansen, Kissei, Pfizer, Sanofi, Takeda, Tanabe-Mitsubishi and UCB. Tsutomu Takeuchi: Honoraria, Astellas Pharma, Bristol-Myers, Chugai Pharmaceutical, Daiichi Sankyo, Takeda Pharmaceutical, Teijin Pharma, AbbVie, Asahikasei Pharma, Mitsubishi Tanabe Pharma, Astra Zeneca, Eli Lilly Japan, Novartis Pharma, Abbivie, Nipponkayaku, Janssen, Pharmaceutical, Taiho Pharmaceutical and Pfizer Japan; Research funding, Astellas Pharma, Bristol-Myers, Chugai Pharmaceutical, Daiichi Sankyo, Takeda Pharmaceutical, Teijin Pharma, AbbVie, Asahikasei Pharma, Mitsubishi Tanabe Pharma, Astra Zeneca, Eli Lilly Japan, Novartis Pharma, Abbivie, Nipponkayaku, Janssen, Pharmaceutical, Taiho Pharmaceutical and Pfizer Japan.

\section{Acknowledgement}

We sincerely thank all the physicians and others who provided care for the patients enrolled in this study.

\section{References}

1. Monnet X, Vidal-Petiot E, Osman D, et al. Critical care management and outcome of severe Pneumocystis pneumonia in patients with and without HIV infection. Crit Care 12: R28, 2008.

2. Akiyama M, Kaneko Y, Takeuchi T. Comparison of the clinical characteristics of Pneumocystis pneumonia between patients with rheumatoid arthritis being treated with biologics and those being treated without biologics. Biomed Res Int 2017: 3710652, 2017.

3. Tasaka S, Tokuda H. Pneumocystis jirovecii pneumonia in nonHIV-infected patients in the era of novel immunosuppressive thera- 
pies. J Infect Chemother 18: 793-806, 2012.

4. Tokuda H, Sakai F, Yamada H, et al. Clinical and radiological features of Pneumocystis pneumonia in patients with rheumatoid arthritis, in comparison with methotrexate pneumonitis and Pneumocystis pneumonia in acquired immunodeficiency syndrome: a multicenter study. Intern Med 47: 915-923, 2008.

5. Harigai M, Koike R, Miyasaka N. Pneumocystis pneumonia associated with infliximab in Japan. N Engl J Med 357: 1874-1876, 2007.

6. Kanda Y. Investigation of the freely available easy-to-use software 'EZR' for medical statistics. Bone Marrow Transplant 48: 452458, 2013.

7. Ranieri VM, Rubenfeld GD, Thompson BT, et al.; ARDS Definition Task Force. Acute respiratory distress syndrome: the Berlin Definition. JAMA 307: 2526-2533, 2012.

8. Kanne JP, Yandow DR, Meyer CA. Pneumocystis jiroveci pneumonia: high-resolution CT findings in patients with and without HIV infection. AJR Am J Roentgenol 198: W555-W561, 2012.

9. $\mathrm{Mu} \mathrm{XD}$, Jia $\mathrm{P}, \mathrm{Gao} \mathrm{L}$, et al. Relationship between radiological stages and prognoses of Pneumocystis pneumonia in non-AIDS immunocompromised patients. Chin Med J (Engl) 129: 20202025, 2016.

10. Kumagai S, Arita M, Koyama T, et al. Prognostic significance of crazy paving ground grass opacities in non-HIV Pneumocystis ji- rovecii pneumonia: an observational cohort study. BMC Pulm Med 19: 47,2019

11. Limper AH, Offord KP, Smith TF, Martin WJ 2nd. Pneumocystis carinii pneumonia. Differences in lung parasite number and inflammation in patients with and without AIDS. Am Rev Respir Dis 140: 1204-1209, 1989.

12. Tasaka S, Tokuda H, Sakai F, et al. Comparison of clinical and radiological features of Pneumocystis pneumonia between malignancy cases and acquired immunodeficiency syndrome cases: a multicenter study. Intern Med 49: 273-281, 2010.

13. Chen M, Tian X, Qin F, et al. Pneumocystis pneumonia in patients with autoimmune diseases: a retrospective study focused on clinical characteristics and prognostic factors related to death. PLoS One 10: e0139144, 2015.

14. Hoeboer SH, Oudemans-van Straaten HM, Groeneveld AB. Albumin rather than $\mathrm{C}$-reactive protein may be valuable in predicting and monitoring the severity and course of acute respiratory distress syndrome in critically ill patients with or at risk for the syndrome after new onset fever. BMC Pulm Med 15: 22, 2015.

The Internal Medicine is an Open Access journal distributed under the Creative Commons Attribution-NonCommercial-NoDerivatives 4.0 International License. To view the details of this license, please visit (https://creativecommons.org/licenses/ by-nc-nd/4.0/).

(C) 2019 The Japanese Society of Internal Medicine Intern Med 58: 3379-3383, 2019 